\title{
Identification of active miRNA and transcription factor regulatory pathways in human obesity-related inflammation
}

\author{
Xi-Mei Zhang ${ }^{1 *+}$, Lin Guo ${ }^{2 \dagger}$, Mei-Hua Chi ${ }^{3}$, Hong-Mei Sun ${ }^{4}$ and Xiao-Wen Chen ${ }^{4}$
}

\begin{abstract}
Background: Obesity-induced chronic inflammation plays a fundamental role in the pathogenesis of metabolic syndrome (MS). Recently, a growing body of evidence supports that miRNAs are largely dysregulated in obesity and that specific miRNAs regulate obesity-associated inflammation. We applied an approach aiming to identify active miRNA-TF-gene regulatory pathways in obesity. Firstly, we detected differentially expressed genes (DEGs) and differentially expressed miRNAs (DEmiRs) from mRNA and miRNA expression profiles, respectively. Secondly, by mapping the DEGs and DEmiRs to the curated miRNA-TF-gene regulatory network as active seed nodes and connect them with their immediate neighbors, we obtained the potential active miRNA-TF-gene regulatory subnetwork in obesity. Thirdly, using a Breadth-First-Search (BFS) algorithm, we identified potential active miRNA-TF-gene regulatory pathways in obesity. Finally, through the hypergeometric test, we identified the active miRNA-TF-gene regulatory pathways that were significantly related to obesity.

Results: The potential active pathways with FDR $<0.0005$ were considered to be the active miRNA-TF regulatory pathways in obesity. The union of the active pathways is visualized and identical nodes of the active pathways were merged.
\end{abstract}

Conclusions: We identified 23 active miRNA-TF-gene regulatory pathways that were significantly related to obesity-related inflammation.

Keywords: Obesity, Inflammation, Adipokines

\section{Background}

The prevalence of obesity has increased drastically worldwide. Overweight represents major health burdens and is associated with a cluster of metabolic disorders, such as insulin resistence and cardiovascular disease [1]. Obesity is associated with a low-grade inflammation, which influences adipocyte function and may promote type 2 diabetes and MS [2,3].

Adipocytes and infiltrating inflammatory cells located within the adipose tissue (AT) secrete a number of bioactive molecules, such as tumor necrosis fator- $\alpha$ (TNF- $\alpha$ ), interleukin (IL)- 6 and adiponectin collectively called adipokines. They are labeled as anti-inflammatory or pre-

\footnotetext{
*Correspondence: ximei1119@126.com

${ }^{\dagger}$ Equal contributors

'Department of Histology and Embryology, Harbin Medical University, Harbin 150081, PR China

Full list of author information is available at the end of the article
}

inflammatory adipokines $[4,5]$. Obesity frequently leads to a dysregulation of adipokine secretion [6,7]. However, the mechanisms controlling adipokines production in obesity are not clear.

In recent years, a lot of evidence has highlighted the modulating roles of miRNAs in inflammatory system $[8,9]$. MiRNAs may act directly on the target genes or indirectly by first regulating transcription factors (TFs), which then control the expression of genes [9]. The role of miRNAs in adipose inflammation and obesity is still not fully settled. Thus, integrated analysis of transcriptional and post- transcriptional regulation could provide a comprehensive regulatory map for the mechanism study of the AT inflammation in obesity.

In this study, by using hypergeometric test we found 23 active post-transcriptional regulatory pathways in obesity based on obesity-related mRNA and miRNA expression profiles. 


\section{Methods}

We applied a novel approach to identify active miRNATF regulatory pathways in obesity. Firstly, we found differentially expressed genes (DEGs) and differentially expressed miRNAs (DEmiRs) from mRNA and miRNA expression profiles which were derived from the Gene Expression Omnibus (GEO) database [10]. Secondly, we map the DEGs and DEmiRs to the curated miRNA-TF regulatory network as active seed nodes and connect them with their immediate neighbor. In this way, the potential active miRNA-TF regulatory subnetworks in obesity were obtained. Thirdly, based on a BFS algorithm, we identified all of the directed acyclic paths between 0 -indegree nodes, where the indegree is 0 , and 0 -outdegree nodes, where the outdegree is 0 . These paths were defined as potential active miRNA-TF regulatory pathways in obesity. Finally, we mapped known obesity-associated miRNAs, TFs and adipokines to potential active pathways. We identified the active miRNATF-gene regulatory pathways that were significantly related to obesity based on the hypergeometric test. An overview of the approach is shown in Figure 1.

\section{Obesity-related mRNA and miRNA expression profiles}

The mRNA and miRNA expression profiles (GSE2508 and GSE18470) of human subcutaneous adipose were downloaded from GEO database (Additional file 1: Table S1 and Additional file 2: Table S2).

For mRNA expression profile, 20 lean and 19 obese subjects were hybridized individually to Affymetrix oligonucleotide arrays HG-U95A, B, C, D, and E 6 platforms. In each platform, probe sets were mapped to Entrez Gene IDs. We averaged the expression values of the probe sets which corresponded to the same gene. The differentially expressed genes were detected using Significance Analysis of Microarrays (SAM) approach [11], and false discovery rate (FDR) was used to correct for multiple testing. The genes which were differentially expressed in all of 6 platforms were considered in the analysis.

For miRNA expression profile, we filtered out samples with Type 2 Diabetes Mellitus. Then 6 lean and 13 obese samples were kept for the further analysis. The differentially expressed miRNAs were detected using SAM.

\section{Curated TF and miRNA regulatory network}

We generated TF and miRNA regulatory network by merging miRTarBase,miRecords, TransmiR,TRANSFAC, and TarBase [12-16]. Here, the curated human TF-genes were derived from the TRANSFAC database. The curated TF-miRNA regulations were derived from TransmiR database. The curated human miRNA-gene regulations were obtained from the union of miRecords, TarBase and

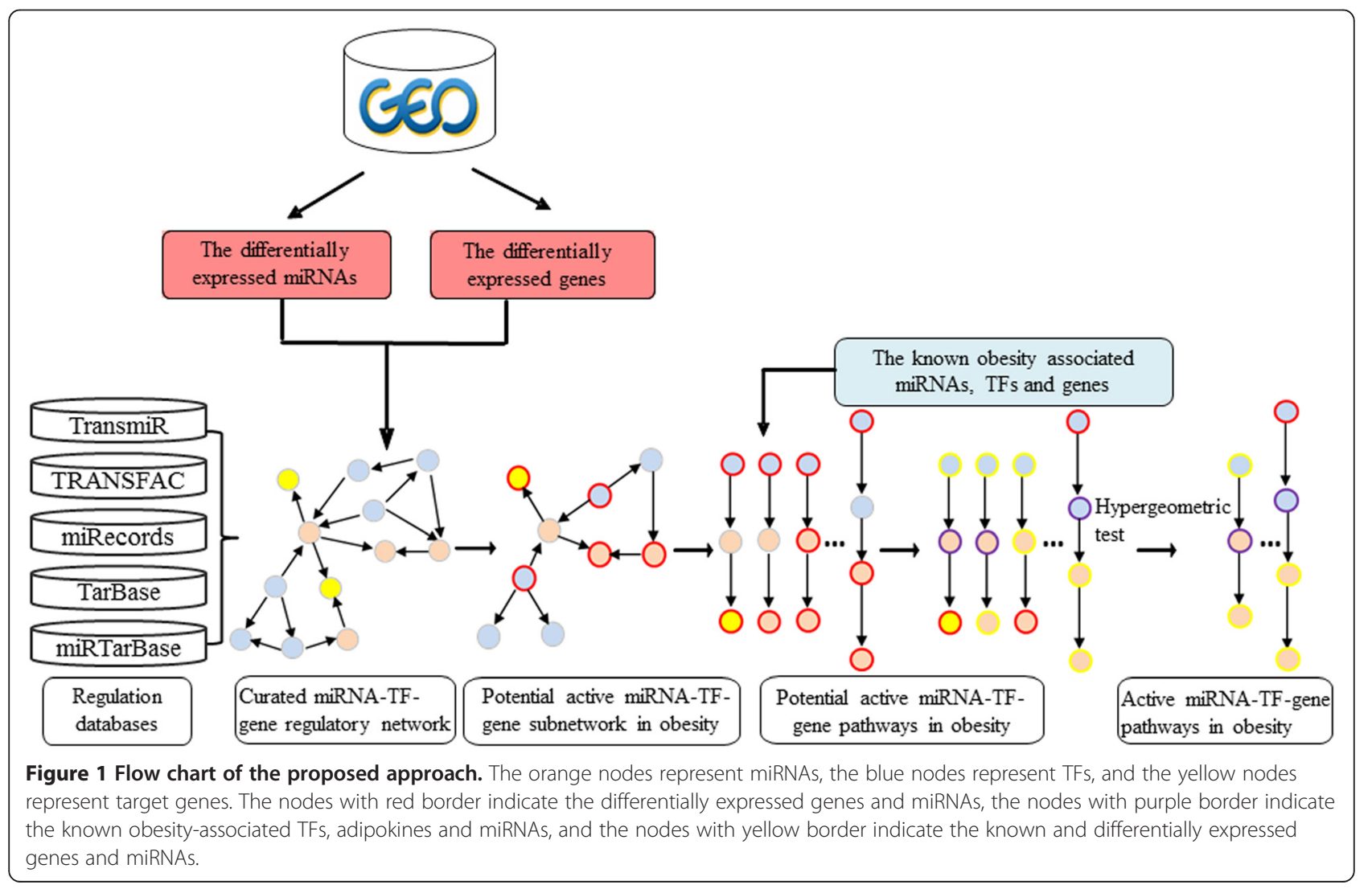


miRTarBase databases. Within the curated regulatory network, we combined all of the redundant edges into a single edge and deleted all of the self-directed edges from the network [17].

\section{Known obesity-associated genes and miRNAs}

The known obesity-associated genes $[18,19]$ and miRNAs $[2,20]$ are manually collected from published studies.

\section{Identification of a potential active miRNA -TF regulatory subnetwork in obesity}

It was reported that some disease-related key genes could hide among non-DE genes [21,22]. So we hypothesized that the DEGs, DEmiRs, and their immediate neighbors in the constructed miRNA-TF regulatory network potentially induced the pathology of obesity. We generated the potential active miRNA-TF regulatory subnetwork by mapping the DEGs and DEmiRs into the regulatory network as active seed nodes and connecting them with their neighbors [17].

\section{Identification of potential active miRNA -TF regulatory pathways in obesity}

Based on the potential active miRNA-TF-gene regulatory subnetwork, all directed acyclic paths from 0-indegree nodes to 0-outdegree nodes were found. The gene/ miRNA with 0 -indegree is located upstream of the regulatory pathway. The gene/miRNA with a 0 -outdegree is located downstream of the regulatory pathway. And the upstream genes/miRNAs' activation could cause a cascade effect which results in the regulation of downstream gene/miRNA expression and leads to obesity disease. In this study, we defined potential active regulatory pathways as the directed acyclic paths with more than 2 nodes as, which contained at least one DE node and no more than one non-DE node or nodes without expression values between the two DE nodes [17].

\section{Evaluation of potential active miRNA-TF-gene regulatory pathways in obesity}

Coverage rate $(C R)$ of known obesity-associated genes and miRNAs in the potential active pathway is used to measure the strength of the relationships between the potential active pathway and obesity. $C R$ was calculated according to

$$
C R=\frac{N_{D}}{N_{T}}
$$

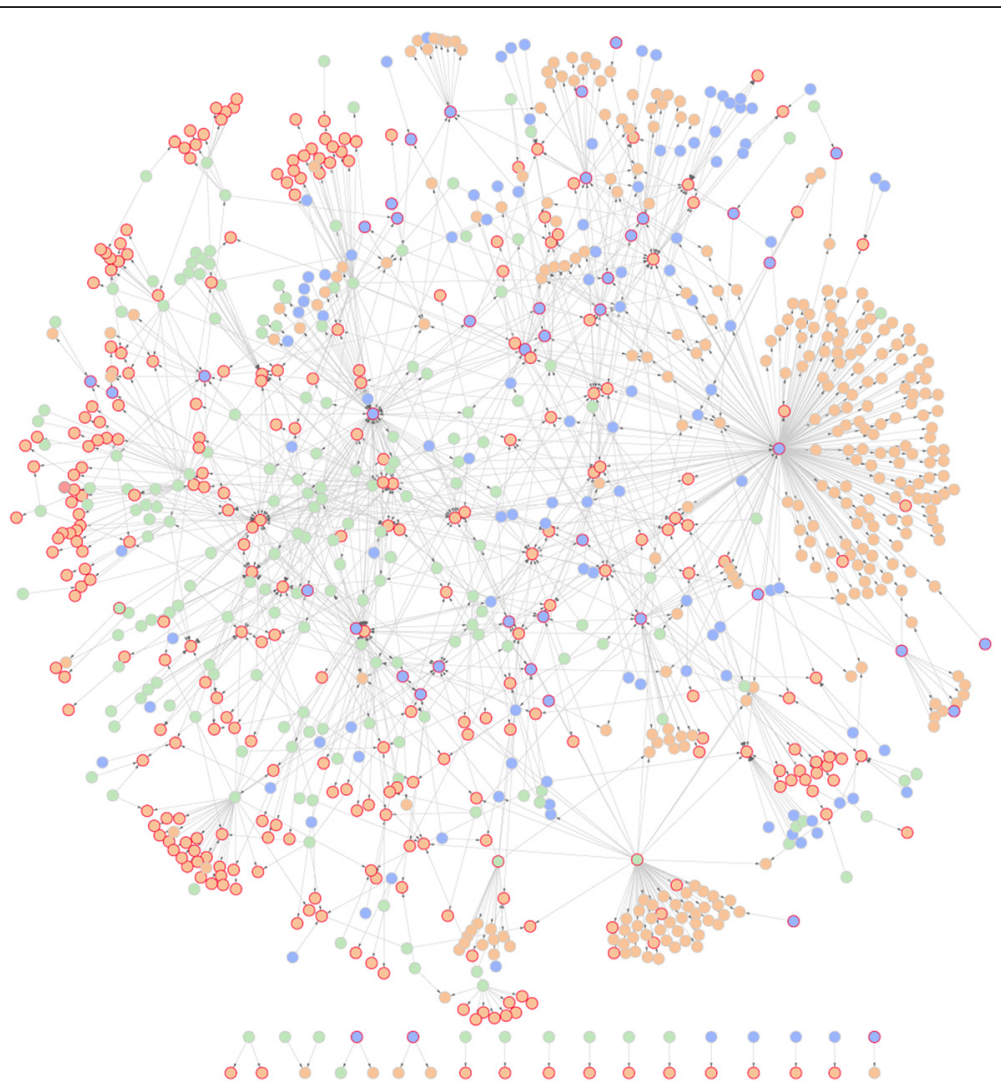

Figure 2 The potential active miRNA-TF-gene regulatory subnetwork in obesity. The orange nodes represent miRNAs, the blue nodes represent TFs, and the green nodes represent target genes. The red border indicates the differentially expressed genes and miRNAs. 
Where $N_{D}$ represents the number of known obesity associated genes and miRNAs in the pathway, and $N_{T}$ represents the total number of genes and miRNAs in the pathway. Then, hypergeometric test were used to evaluate the statistical significance of $C R$ value. We further adjusted $p$ values for multiple testing using FDR [17].

\section{Results}

Potential active miRNA -TF regulatory subnetwork in obesity We identified 1650 DEGs using FDR $<0.01$ as threshold and 14 DEmiRs with $p$-value $\leq 0.05$. The transcriptional and post-transcriptional regulations were obtained by integrating from TRANSFAC, TransmiR, miRTarBase, miRecords and TarBase to construct the curated miRNA-TF regulatory network [17]. Then the DEGs and DEmiRs were mapped to the curated miRNA-TF-gene regulatory network as active seeds. We constructed the potential active miRNATF-gene regulatory subnetwork by connecting all of the active seeds with their immediate neighbors (Figure 2). Finally, the subnetwork comprised 345 nodes and 1379 edges, in which 1661 genes and 3 miRNAs were differentially expressed.

The active miRNA-TF-gene regulatory pathways in obesity We identified all of the directed acyclic paths from 0 -indegree nodes to 0 -outdegree nodes in the potential active miRNA-TF-gene regulatory subnetwork by BFS approach. As a result, 328800 paths with more than 2 nodes were obtained, which were regarded as the potential active miRNA-TF regulatory pathways in obesity. These pathways

Table 1 Active miRNA-TF-gene regulatory pathways in obesity

\begin{tabular}{|c|c|c|c|c|c|}
\hline Active TF-miRNA regulatory pathway & $\begin{array}{l}\text { Number of known AD } \\
\text { genes and miRNAs }\end{array}$ & Pathway length & CR value & p-value & FDR \\
\hline hsa-miR-193b $\rightarrow$ ETS1 $\rightarrow$ TNF-a & 3 & 3 & 1 & 0 & 0 \\
\hline hsa-miR-193b $\rightarrow$ ETS1 $\rightarrow$ NFKB1 & 3 & 3 & 1 & 0 & 0 \\
\hline $\mathrm{A} \rightarrow$ FLI1 $\rightarrow$ hsa-let-7a $\rightarrow$ MYC $\rightarrow$ hsa-miR-20b $\rightarrow$ STAT3 $\rightarrow$ B $\rightarrow$ TNF-a & 8 & 15 & 0.533 & $8.78 \mathrm{E}-10$ & 0.000486716 \\
\hline $\mathrm{A} \rightarrow \mathrm{MYC} \rightarrow$ hsa-miR-29b $\rightarrow \mathrm{SP} 1 \rightarrow \mathrm{TP} 53 \rightarrow \mathrm{EGFR} \rightarrow \mathrm{B} \rightarrow \mathrm{TNF}-\mathrm{a}$ & 8 & 15 & 0.533 & $8.78 \mathrm{E}-10$ & 0.000486716 \\
\hline $\mathrm{A} \rightarrow \mathrm{FLI1} \rightarrow$ hsa-let-7a $\rightarrow$ MYC $\rightarrow$ hsa-miR-20b $\rightarrow$ STAT3 $\rightarrow$ B $\rightarrow$ NFKB1 & 8 & 15 & 0.533 & $8.78 \mathrm{E}-10$ & 0.000486716 \\
\hline $\mathrm{A} \rightarrow \mathrm{MYC} \rightarrow$ hsa-miR-29b $\rightarrow \mathrm{SP} 1 \rightarrow \mathrm{TP} 53 \rightarrow \mathrm{EGFR} \rightarrow \mathrm{B} \rightarrow \mathrm{NFKB} 1$ & 8 & 15 & 0.533 & $8.78 \mathrm{E}-10$ & 0.000486716 \\
\hline $\mathrm{C} \rightarrow$ hsa-miR-29b $\rightarrow \mathrm{SP} 1 \rightarrow \mathrm{TNF}$ & 8 & 15 & 0.533 & $8.78 \mathrm{E}-10$ & 0.000486716 \\
\hline $\mathrm{C} \rightarrow$ hsa-miR-29b $\rightarrow$ SP1 $\rightarrow$ RELA & 8 & 15 & 0.533 & $8.78 \mathrm{E}-10$ & 0.000486716 \\
\hline$C \rightarrow$ hsa-miR-22 $\rightarrow$ MAX $\rightarrow$ hsa-miR-193a & 8 & 15 & 0.533 & $8.78 \mathrm{E}-10$ & 0.000486716 \\
\hline $\mathrm{C} \rightarrow$ hsa-miR-29b $\rightarrow \mathrm{SP} 1 \rightarrow \mathrm{RBP} 4$ & 8 & 15 & 0.533 & $8.78 \mathrm{E}-10$ & 0.000486716 \\
\hline $\mathrm{D} \rightarrow \mathrm{FLT1} \rightarrow$ hsa-let-7a & 8 & 15 & 0.533 & $8.78 \mathrm{E}-10$ & 0.000486716 \\
\hline $\mathrm{C} \rightarrow$ hsa-miR-29b $\rightarrow$ SP1 $\rightarrow$ VEGFA & 8 & 15 & 0.533 & $8.78 \mathrm{E}-10$ & 0.000486716 \\
\hline $\mathrm{C} \rightarrow$ hsa-miR-29b $\rightarrow$ SP1 $\rightarrow$ SERPINE1 & 8 & 15 & 0.533 & $8.78 \mathrm{E}-10$ & 0.000486716 \\
\hline $\mathrm{C} \rightarrow$ hsa-miR-29b $\rightarrow$ SP1 $\rightarrow$ REL & 8 & 15 & 0.533 & $8.78 \mathrm{E}-10$ & 0.000486716 \\
\hline C $\rightarrow$ hsa-miR-29b $\rightarrow$ SP1 $\rightarrow$ CCL2 & 8 & 15 & 0.533 & $8.78 \mathrm{E}-10$ & 0.000486716 \\
\hline $\mathrm{D} \rightarrow \mathrm{STAT} 1 \rightarrow \mathrm{CCL} 2$ & 8 & 15 & 0.533 & $8.78 \mathrm{E}-10$ & 0.000486716 \\
\hline $\mathrm{C} \rightarrow$ hsa-miR-29b $\rightarrow$ SP1 $\rightarrow$ TP53 & 8 & 15 & 0.533 & $8.78 \mathrm{E}-10$ & 0.000486716 \\
\hline $\mathrm{E} \rightarrow \mathrm{TNF}$ & 8 & 15 & 0.533 & $8.78 \mathrm{E}-10$ & 0.000486716 \\
\hline $\mathrm{E} \rightarrow \mathrm{NFKB} 1$ & 8 & 15 & 0.533 & $8.78 \mathrm{E}-10$ & 0.000486716 \\
\hline $\mathrm{F} \rightarrow$ FLI1 $\rightarrow$ hsa-let-7a $\rightarrow$ MYC $\rightarrow$ hsa-miR-20b $\rightarrow$ STAT3 $\rightarrow$ G $\rightarrow$ TNF & 8 & 15 & 0.533 & $8.78 \mathrm{E}-10$ & 0.000486716 \\
\hline $\mathrm{F} \rightarrow \mathrm{MYC} \rightarrow$ hsa-miR-29b $\rightarrow \mathrm{SP} 1 \rightarrow \mathrm{TP} 53 \rightarrow \mathrm{EGFR} \rightarrow \mathrm{G} \rightarrow \mathrm{TNF}$ & 8 & 15 & 0.533 & $8.78 \mathrm{E}-10$ & 0.000486716 \\
\hline $\mathrm{F} \rightarrow \mathrm{FLI1} \rightarrow$ hsa-let-7a $\rightarrow$ MYC $\rightarrow$ hsa-miR-20b $\rightarrow$ STAT3 $\rightarrow$ G $\rightarrow$ NFKB1 & 8 & 15 & 0.533 & $8.78 \mathrm{E}-10$ & 0.000486716 \\
\hline $\mathrm{F} \rightarrow \mathrm{MYC} \rightarrow$ hsa-miR-29b $\rightarrow \mathrm{SP} 1 \rightarrow \mathrm{TP} 53 \rightarrow \mathrm{EGFR} \rightarrow \mathrm{G} \rightarrow \mathrm{NFKB} 1$ & 8 & 15 & 0.533 & $8.78 \mathrm{E}-10$ & 0.000486716 \\
\hline
\end{tabular}

A for hsa-miR-204 $\rightarrow$ SNAl2 $\rightarrow$ hsa-miR-200c $\rightarrow$ JAG1 $\rightarrow$ hsa-miR-145.

B for hsa-miR-21 $\rightarrow$ IL-1 $\beta \rightarrow$ hsa-miR-9 $\rightarrow$ ETS1.

C for SPI $\rightarrow$ IL1B $\rightarrow$ hsa-miR-9 $\rightarrow$ ETS1 $\rightarrow$ has-miR-146a $\rightarrow$ EGFR $\rightarrow$ hsa-miR-21 $\rightarrow$ JAG1 $\rightarrow$.

hsa-miR-145 $\rightarrow$ FLI1 $\rightarrow$ hsa-let-7a $\rightarrow$ MYC.

D for SPI1 $\rightarrow$ IL1B $\rightarrow$ hsa-miR-9 $\rightarrow$ ETS1 $\rightarrow$ TFAP2A $\rightarrow$ MYC $\rightarrow$ hsa-miR-29b $\rightarrow$ SP1 $\rightarrow$ TP53 $\rightarrow$.

EGFR $\rightarrow$ hsa-miR-21 $\rightarrow$ JAG1 $\rightarrow$ hsa-miR-145.

E for TP63 $\rightarrow$ JAG1 $\rightarrow$ hsa-miR-145 $\rightarrow$ FLI1 $\rightarrow$ hsa-let-7a $\rightarrow$ MYC $\rightarrow$ hsa-miR-29b $\rightarrow$ SP1 $\rightarrow$ TP53 $\rightarrow$.

EGFR $\rightarrow$ hsa-miR-21 $\rightarrow$ IL1B $\rightarrow$ hsa-miR-9 $\rightarrow$ ETS1.

F for hsa-miR-124 $\rightarrow$ SNAI2 $\rightarrow$ hsa-miR-200c $\rightarrow$ JAG1 $\rightarrow$ hsa-miR-145.

G forhsa-miR-21 $\rightarrow$ IL1B $\rightarrow$ hsa-miR-9 $\rightarrow$ ETS1. 
contained 568 genes and miRNAs. The length of all of the potential active pathways ranged from 3 to 15 , and the average was 11.67.

Furthermore, we derived 34 known obesity-associated genes, $29 \mathrm{TFs}$ and 11miRNAs to evaluate the association between the identified potential active pathways and obesity. There were 41 obesity-associated genes and miRNAs mapped in the potential active pathways. The coverage rate $(C R)$ of the known obesity-associated genes and miRNAs of the potential active pathway was used to measure the strength of the association between the potential active pathway and obesity. Next, we identified the significantly active pathways using a hypergeometric test. The potential active pathways with FDR $<0.0005$ were considered to be the active miRNA-TF regulatory pathways in obesity. As a result, we identified 23 active pathways (Table 1). The union of the 23 active pathways is visualized in Figure 3, and identical nodes of the active pathways were merged.

\section{Discussion}

Evidence has indicated that miRNAs are largely dysregulated in obesity and that specific miRNAs regulate obesityassociated inflammation [20]. In this study, we proposed a novel approach to identify active miRNA-TF-gene regulatory pathways by integrating obesity-related mRNA and miRNA expression profiles and transcriptional and post-transcriptional regulation. As a result, we identified 23 active miRNA-TF-gene regulatory pathways that were significantly related to obesity.

In these 23 pathways, 6 adipokines including IL-1 $\beta$, $\mathrm{CCL}_{2}$, RBP4, VEGFA,SERPINE4 and TNF- $\alpha$ are involved. IL- $1 \beta$ is regulated by TF SPI1 and has-miR- 21 . The has-miR-21 is involved in the complex regulation subnet. IL-1 $\beta$ is expressed in and secreted from adipose tissue [23]. IL-1 $\beta$ is a proinflammatory cytokine which has been proposed to play a role in inflammatory pancreatic $\beta$-cell destruction leading to type 1diabetes [24]. Obesity is associated with a low-grade inflammatory state in white adipose tissue (WAT). Adipocytes and infiltrating inflammatory cells (primarily macrophages) present within the tissue secrete key inflammatory proteins, such as TNF-a, IL-6, and $\mathrm{CCL}_{2} /$ monocyte chemoattractant protein, and their gene expression and release are markedly increased in obesity $[4,5] . \mathrm{CCL}_{2}$ has been proposed to initiate adipose inflammation by attracting inflammatory cells from the blood stream into WAT and is essential for the development of WAT inflammation [25-27]. The miR-126 reduces the production of $\mathrm{CCL}_{2}$ by targeting directly the 3 '-UTR of $\mathrm{CCL}_{2}$, while miR193b inhibited indirectly the $\mathrm{CCL}_{2}$ production through down regulating the transcription factors of $\mathrm{CCL}_{2}$ (RELB, STAT6, and ETS1) [2]. In our detected subnet, TF STAT1 and SP1 directly regulate the expression of $\mathrm{CCL}_{2}$, and hsa-miR-145, has- miR-29b through these 2 TFs make regulation on $\mathrm{CCL}_{2}$. VEGFA stimulates angiogenesis in adipose tissue,SERPINE4 belongs to proinflammatory adipokines, and RBP4 is associated with insulin resistance and visceral fat distribution [18]. They are also regulated by SP1 directly and by has- miR-29b through SP1 indirectly. TNF- $\alpha$ is recognized as a multifunctional cytokine implicated in inflammation, apoptosis and cell survival as well as induction of insulin resistance [28-31]. By use of motif activity response analysis, we were able to dissect pathways linking miRNAs, TFs, and TNF- $\alpha$. The predicted network comprised several TFs with well-characterized roles in obesity-induced inflammation and three miRNAs that were directly or indirectly linked to TNF- $\alpha$. For one of the miRNAs, we were able to confirm the regulatory pathways proposed by our model. In particular, we demonstrated a signal circuit from has-miR-193b to TNF- $\alpha$ either via ETS1 as

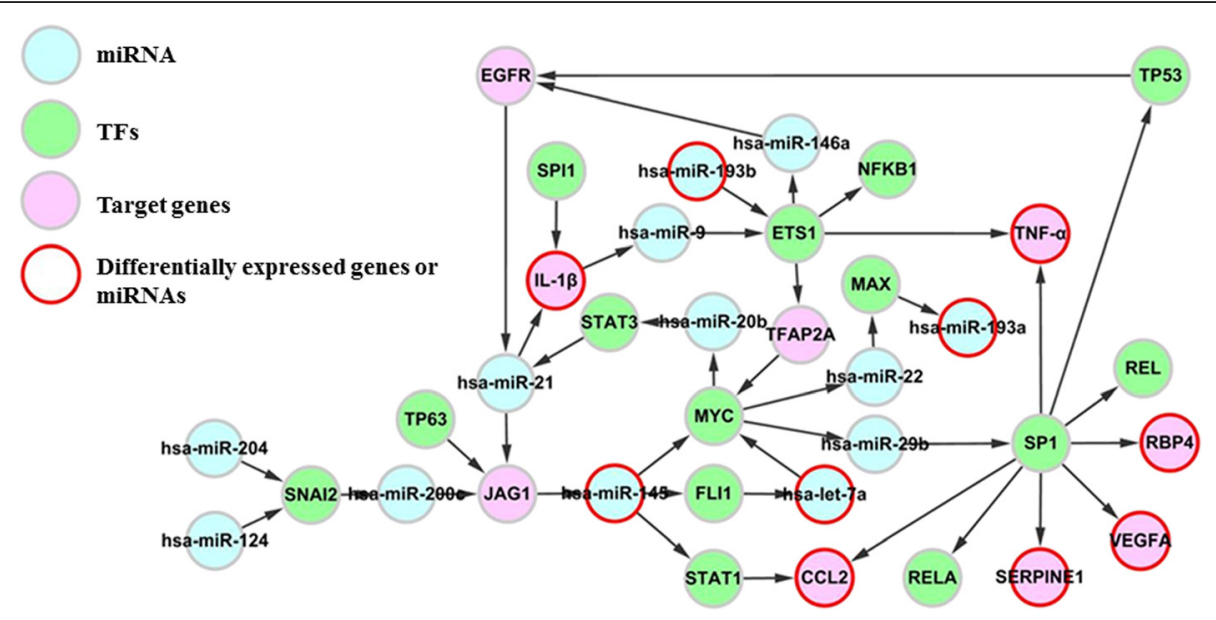

Figure 3 Union of 23 active miRNA-TF-gene regulatory pathways in obesity. 
a single TF step. MiRNA-193b may be of particular importance since its expression was associated with TNF- $\alpha$ mRNA expression. Evidence has provided that specific miRNAs regulate the polarization of macrophages and the expression and secretion of adipokines in WAT and a subset of miRNAs could be packaged into adipocytederived microvesicles and delivered into blood or neighboring cells, probably acting as inflammatory communicators between adipocytes, macrophages, and distant cells [20]. MiRNAs are secreted into circulation in a stable manner and correlated closely with crucial metabolic parameters, the plasma miRNA profiles could therefore be used as attractive biomarkers for the metabolic syndrome (MS) $[20,32,33]$. However, whether the signaling circuit established in circulation is also present in adipocytes remains to be determined.

Taken together, our results suggest that specific miRNAs may be important regulators of inflammation in human circulation through their effects on adipokines levels. This may be mediated by indirect effects on TF circuits. miRNAs could therefore constitute novel potential targets for treating obesity comorbidities.

\section{Conclusions}

We identified 23 active miRNA-TF-gene regulatory pathways that were significantly related to obesity-related inflammation.

\section{Additional files}

Additional file 1: Table S1. Obesity-related mRNA expression profiles in adipocytes of obese humans.

Additional file 2: Table S2. Obesity-related miRNA expression profiles in human adipose tissue.

\section{Competing interests}

The authors declare that they have no competing interests.

\section{Authors' contributions}

X-MZ conceived and carried out experiments. LG and M-HC carried out experiments. H-MS analysed data. X-WC helped to draft the manuscript. All authors read and approved the final approval manuscript.

\section{Acknowledgements}

This work was supported by the Natural Science Foundation of China (Grant No. 81302420); a grant from the 49th China Postdoctoral Funds (20110491103); the Heilongjiang Postdoctoral Funds (LRB-2010-338); the Science Foundation of Heilongjiang Province Health Department (2012-806). We thank all the research staff for their contributions to this project and the anonymous reviewers for their helpful comments, which have greatly improved the manuscript.

\section{Author details}

${ }^{1}$ Department of Histology and Embryology, Harbin Medical University, Harbin 150081, PR China. ${ }^{2}$ Department of Endocrinology and Metabolism, the Second Affiliated Hospital of Harbin Medical University, Harbin 150081, PR China. ${ }^{3}$ Teaching Experiment Center of Morphology, Harbin Medical University, Harbin 150081, PR China. ${ }^{4}$ College of Bioinformatics Science and Technology, Harbin Medical University, Harbin 150081, PR China.
Received: 11 November 2014 Accepted: 24 February 2015

Published online: 07 March 2015

\section{References}

1. Tseng YH, Cypess AM, Kahn CR. Cellular bioenergetics as a target for obesity therapy. Nat Rev Drug Discov. 2010;9(6):465-82.

2. Arner E, Mejhert N, Kulyte A, Balwierz PJ, Pachkov M, Cormont M, et al. Adipose tissue microRNAs as regulators of CCL2 production in human obesity. Diabetes. 2012;61(8):1986-93.

3. Hotamisligil GS. Inflammation and metabolic disorders. Nature. 2006;444(7121):860-7.

4. Ouchi N, Parker JL, Lugus JJ, Walsh K. Adipokines in inflammation and metabolic disease. Nat Rev Immunol. 2011;11(2):85-97.

5. Fain JN. Release of inflammatory mediators by human adipose tissue is enhanced in obesity and primarily by the nonfat cells: a review. Mediators Inflamm. 2010;2010:513948.

6. Bluher M. Adipose tissue dysfunction in obesity. Exp Clin Endocrinol Diabetes. 2009;117(6):241-50.

7. Catalan V, Gomez-Ambrosi J, Rodriguez A, Salvador J, Fruhbeck G. Adipokines in the treatment of diabetes mellitus and obesity. Expert Opin Pharmacother. 2009;10(2):239-54.

8. Lu LF, Liston A. MicroRNA in the immune system, microRNA as an immune system. Immunology. 2009;127(3):291-8.

9. Sayed D, Abdellatif M. MicroRNAs in development and disease. Physiol Rev. 2011;91(3):827-87.

10. Edgar R, Domrachev M, Lash AE. Gene Expression Omnibus: NCBI gene expression and hybridization array data repository. Nucleic Acids Res. 2002;30(1):207-10.

11. Tusher VG, Tibshirani R, Chu G. Significance analysis of microarrays applied to the ionizing radiation response. Proc Natl Acad Sci U S A. 2001;98 (9):5116-21.

12. Wingender $E$, Chen $X$, Hehl R, Karas H, Liebich I, Matys V, et al. TRANSFAC: an integrated system for gene expression regulation. Nucleic Acids Res. 2000;28(1):316-9.

13. Hsu SD, Lin FM, Wu WY, Liang C, Huang WC, Chan WL, et al. miRTarBase: a database curates experimentally validated microRNA-target interactions. Nucleic Acids Res. 2011;39(Database issue):D163-169.

14. Xiao F, Zuo Z, Cai G, Kang S, Gao X, Li T. miRecords: an integrated resource for microRNA-target interactions. Nucleic Acids Res. 2009;37(Database issue): D105-110.

15. Wang J, Lu M, Qiu C, Cui Q. TransmiR: a transcription factor-microRNA regulation database. Nucleic Acids Res. 2010;38(Database issue):D119-122.

16. Sethupathy P, Corda B, Hatzigeorgiou AG. TarBase: A comprehensive database of experimentally supported animal microRNA targets. RNA. 2006;12(2):192-7

17. Jiang W, Zhang Y, Meng F, Lian B, Chen X, Yu X, et al. Identification of active transcription factor and miRNA regulatory pathways in Alzheimer's disease. Bioinformatics. 2013;29(20):2596-602.

18. Bluher M. Adipokines - removing road blocks to obesity and diabetes therapy. Mol Metab. 2014;3(3):230-40.

19. Jung UJ, Choi MS. Obesity and its metabolic complications: the role of adipokines and the relationship between obesity, inflammation, insulin resistance, dyslipidemia and nonalcoholic fatty liver disease. Int J Mol Sci. 2014;15(4):6184-223.

20. Ge Q, Brichard $S, Y i$ X, Li Q. microRNAs as a new mechanism regulating adipose tissue inflammation in obesity and as a novel therapeutic strategy in the metabolic syndrome. J Immunol Res. 2014;2014:987285.

21. Nitsch D, Tranchevent LC, Thienpont B, Thorrez L, Van Esch H, Devriendt K, et al. Network analysis of differential expression for the identification of disease-causing genes. PLoS One. 2009;4(5):e5526.

22. Zhao J, Yang TH, Huang Y, Holme P. Ranking candidate disease genes from gene expression and protein interaction: a Katz-centrality based approach. PLoS One. 2011;6(9):e24306.

23. Sopasakis VR, Nagaev I, Smith U. Cytokine release from adipose tissue of nonobese individuals. Int J Obes (Lond). 2005;29(9):1144-7.

24. Larsen CM, Faulenbach M, Vaag A, Volund A, Ehses JA, Seifert B, et al. Interleukin-1-receptor antagonist in type 2 diabetes mellitus. N Engl J Med. 2007;356(15):1517-26.

25. Anderson EK, Gutierrez DA, Hasty AH. Adipose tissue recruitment of leukocytes. Curr Opin Lipidol. 2010;21(3):172-7.

26. Deshmane SL, Kremlev S, Amini S, Sawaya BE. Monocyte chemoattractant protein-1 (MCP-1): an overview. J Interferon Cytokine Res. 2009;29(6):313-26. 
27. Kanda H, Tateya S, Tamori Y, Kotani K, Hiasa K, Kitazawa R, et al. MCP-1 contributes to macrophage infiltration into adipose tissue, insulin resistance, and hepatic steatosis in obesity. J Clin Invest. 2006;116(6):1494-505.

28. Hotamisligil GS, Spiegelman BM. Tumor necrosis factor alpha: a key component of the obesity-diabetes link. Diabetes. 1994;43(11):1271-8.

29. Hotamisligil GS, Shargill NS, Spiegelman BM. Adipose expression of tumor necrosis factor-alpha: direct role in obesity-linked insulin resistance. Science. 1993;259(5091):87-91.

30. Hotamisligil GS, Arner P, Caro JF, Atkinson RL, Spiegelman BM. Increased adipose tissue expression of tumor necrosis factor-alpha in human obesity and insulin resistance. J Clin Invest. 1995;95(5):2409-15.

31. Uysal KT, Wiesbrock SM, Marino MW, Hotamisligil GS. Protection from obesity-induced insulin resistance in mice lacking TNF-alpha function. Nature. 1997;389(6651):610-4

32. Heneghan HM, Miller N, McAnena OJ, O'Brien T, Kerin MJ. Differential miRNA expression in omental adipose tissue and in the circulation of obese patients identifies novel metabolic biomarkers. J Clin Endocrinol Metab. 2011;96(5):E846-850.

33. Zampetaki A, Kiechl S, Drozdov I, Willeit P, Mayr U, Prokopi M, et al. Plasma microRNA profiling reveals loss of endothelial miR-126 and other microRNAs in type 2 diabetes. Circ Res. 2010;107(6):810-7.

\section{Submit your next manuscript to BioMed Central and take full advantage of:}

- Convenient online submission

- Thorough peer review

- No space constraints or color figure charges

- Immediate publication on acceptance

- Inclusion in PubMed, CAS, Scopus and Google Scholar

- Research which is freely available for redistribution 